\title{
STIMULATORY EFFECT OF ACUTE SINGLE DOSE OF DRIED WHOLE COFFEE CHERRY POWDER ON NRF2 ACTIVITY IN FRESHLY ISOLATED BLOOD CELLS. A SINGLE-BLIND, PLACEBO CONTROLLED CROSS-OVER PILOT CLINICAL STUDY
}

\author{
T. Reyes-Izquierdo ${ }^{1}$, B. Nemzer ${ }^{2}$, R. Argumedo ${ }^{1}$, M. Cervantes ${ }^{1}$, Z. Pietrzkowski ${ }^{1}$
}

\begin{abstract}
Background: NF-E2-related factor 2 (Nrf2) is a transcription factor that participates in the regulation of antioxidant expression during increased oxidant stress. Several phytochemicals and food products have shown to trigger Nrf2 activity. In this pilot placebo-controlled study the effects of a single dose of dried whole coffee cherry powder ("WCCP") on Nrf2 levels were tested. Objectives: To characterize a blend of WCCP and evaluate the potential effects on mTOR and Nrf2 in healthy subjects. Design: In this cross over study, subjects were given placebo or a single dose of 1000mg WCCP on day 1 and 2 . Blood was collected for four time points. Participants served as their own controls. Setting: After supplementation, blood samples were processed for mTOR and Nrf2 analysis. Blood ATP, glucose and lactate were also measured. Participants: Ten healthy subjects, ages ranging from 22 to 35 years and BMI ranging from 24.1 to $30 \mathrm{~kg} / \mathrm{m}^{2}$ were selected to participate. Results: One $1000 \mathrm{mg}$ dose of WCCP resulted in an average $44 \%$ increase of NRf2 levels 180 minutes after ingestion ( $\mathrm{p}=0.03$ ). Phosphorylated mTOR (Ser 2448) was reduced at 180 minutes after supplement; when compared to placebo. Correlation ("Corr") analyses revealed that increases in Nrf2 appear to be associated with mTOR reduction. Blood glucose and extracellular ATP levels were not changed. Conclusions: WCCP increased Nrf2 3 hours after ingestion. Additional testing is required to verify the potency of WCCP on Nrf2, as well as any potential correlation between mTOR (S2448) reduction and increased levels of Nrf2 after supplementation.
\end{abstract}

Key words: Nrf2 activation, mTOR, dried whole coffee cherry powder, antioxidants.

\section{Introduction}

Nuclear factor NF-E2-related factor 2 (Nrf2) is a key transcription factor in the regulation of antioxidant expression during increased oxidant stress $(1,2)$. This key cell-defense gene regulates the expression of cytoprotective proteins that detoxify harmful cellular compounds, neutralizes reactive oxygen species, directly or indirectly modulates the inflammatory response and immune system, and assists in the repair or removal of damaged macromolecules (2-5). Increased Nrf2 expression has previously been associated with improvements in neurodegenerative, autoimmune, diabetic and renal disease in animal and in vitro models (6).

Activation of Nrf2 and phosphoinositide 3-kinase (PI3K), serine-threonine kinase (AKT), and the

1. Futureceuticals Inc.; 16259 Laguna Canyon Rd, Ste 150, Irvine, CA USA 92618; 2. Futureceuticals Inc.; 2692 N. State Rt. 1-17., Momence, IL, USA 60954

Corresponding Author: Tania Reyes-Izquierdo, 16259 Laguna Canyon Rd Ste 150, Irvine, CA, 92618 USA, Phone +1 949502 4496, Fax +1 949502 4987, Email: treyes@futureceuticals.com

Received April 8, 2016

Accepted for publication April 18, 2016 mammalian target of rapamycin (mTOR) signaling pathways, (also known as PI3K)/AKT/mTOR signaling pathways, respectively), have been observed in certain human cancers and it has been postulated that said activations may be central to tumor development and progression (7). Increased expression of Nrf2 and decreased expression of mTOR has been associated with diminished frequency of tumor development and with smaller tumors in animal models (7). More interestingly, inhibition of PI3K)/AKT/mTOR has been associated with extended life spans in insects, invertebrates, and mammals (8-10). Collectively, these pathways present an attractive target for human longevity investigations.

Polyphenols have been suggested as potential therapeutic compounds that may have a positive effect on several pathological conditions such as neurodegenerative diseases, diabetes, certain cancers and cardiovascular diseases $(11,12)$. Dietary polyphenols have been reported to induce the expression of enzymes involved in cellular antioxidant defenses (13).

Several phytochemicals and food-derived products have similarly been shown to trigger Nrf2 activity (2, 14). Green coffee beans have been reported to contain 
large amounts of polyphenolic antioxidants, such as chlorogenic, caffeic, ferulic, and n-coumarinic acids (15).

Here we have evaluated the potential effects of the novel antioxidant activities inherent in dried whole cherries from the coffee plant on mTOR and Nrf2 expression in healthy humans as a preliminary study on potential for coffee cherry to support human health.

\section{Materials and Methods}

\section{Materials}

5-O-caffeoylquinic acid, (-)-epicatechin, procyanidin dimer B2, quercetin-3-glucoside and rutin were obtained from AASC Ltd (Southampton, UK). Methanol and acetonitrile were obtained from Rathburn Chemicals (Walkburn, Scotland). Formic acid was obtained from Fisher Scientific (Loughborough, UK). Dried, ground whole coffee cherry powder samples, commercially marketed as "CoffeeBerry ${ }^{\circledR}$ Brand whole coffee fruit" was obtained from FutureCeuticals, Inc. (Momence, IL USA). Primary caffeine standard was obtained from USP (Rockville, MD). Perchloric acid (HPLC grade) and acetonitrile (HPLC grade) were purchased from SigmaAldrich Corp. (St. Louis, MO, USA). Primary Sorbent Amine (PSA) was obtained from Supelco Inc. (Bellefonte, PA, USA).

Dulbecco's phosphate buffered saline (PBS) and water were purchased from Sigma-Aldrich Corp. Co. (St Louis, MO, USA). Low protein binding microtubes were obtained from Eppendorf (Hauppauge, NY, USA) and RC DC Protein Assay Kit II was from Bio-Rad (Hercules, CA, USA). ATP-luciferase assays were obtained from EMD Millipore (Billerica, MA, USA). Heparin capillary blood collection tubes were obtained from Safe-TFill ${ }^{\circledR}$ (Ram Scientific Inc. Yonkers, NY). Accutrend ${ }^{\circledR}$ Lactate Point of Care and BM-Lactate Strips ${ }^{\circledR}$ were from Roche (Mannheim, Germany). Accu-Chek ${ }^{\circledR}$ Compact Plus glucometer and Accu-Chek ${ }^{\circledR}$ test strips were from Roche Diagnostics (Indianapolis, IN, USA). TransAM® Nrf2 detection assay was from Active Motif (Carlsbad, CA, USA). Phospho m-TOR (Ser 2448), phospho m-TOR (Ser 2481) and total $\mathrm{m}$-TOR were from Cell Signaling Technologies (Danvers, MA, USA).

\section{Chemistry Analyses}

Chlorogenic acids, procyanidins, flavanols and flavonols of WCCP were characterized by LC-MS (n) and quantified by UV absorbance $(16,17)$. Analysis was carried out on a Thermo Acella HPLC system comprising of an auto-sampler with sampler cooler maintained at $6^{\circ} \mathrm{C}$, an Accela photodiode array (PDA) detector (Thermo Fisher Scientific, San Jose, CA, USA) scanning from 200$600 \mathrm{~nm}$. Samples (5 or 10 $\mu \mathrm{l}$ ) were injected onto a $150 \mathrm{x}$ 3.0mm C18 Accucore (Thermo Fisher Scientific, Waltham, MA, USA) maintained at $40^{\circ} \mathrm{C}$ and eluted with a 5-10-
$50 \%$ gradient of $1.0 \%$ formic acid and acetonitrile at 700 $\mu \mathrm{L} / \mathrm{min}$ over 0-10-20 minutes. After passing through the absorbance detector, the eluant was split, and 200 $\mathrm{uL} / \mathrm{min}$ was directed to the electrospray interface of an ExactiveTM Orbitrap mass spectrometer (Thermo Fisher Scientific, Waltham, MA USA). Samples were run in negative ionization mode, and the scan range was from 150 to $1200 \mathrm{amu}$ with resolution set to 60,000 . Peak identifications were based on co-chromatography with authentic standards, when available, as well as absorbance spectra and published MS2 mass spectra data.

Quantification of hydroxycinnamic compounds was obtained by comparison to an authentic standard of 5-O-caffeoylquinic acid, range 5 to $750 \mathrm{ng}$ at 325 $\mathrm{nm}$, and caffeine at $275 \mathrm{~nm}$ in the range 5 to $750 \mathrm{ng}$. Quantification of minor phenolic compounds was by exact mass measurements of calibration standards over the range of 0.5 to $50 \mathrm{ng}$ using (-)-epicatechin for flavan3-ol monomers and procyanidin B2 for dimer and trimer flavan-3-ols. Quercetin-3-glucoside and quercetin3-rutinoside were quantified as quercetin-3-glucoside equivalents.

The caffeine and trigonelline contents were characterized by HPLC (Agilent 1100; Agilent Technologies, Palo Alto, CA, USA) equipped with diode array detector and quantified by UV absorbance (17, 18). For caffeine testing about $10 \mathrm{mg}$ aliquot of caffeine primary standard was accurately weighed into a $50 \mathrm{~mL}$ volumetric flask. The volume was made up to $25 \mathrm{~mL}$ with mobile phase $(90 \%$ of $0.1 \%$ perchloric acid and $10 \%$ acetonitrile) and sonicated for $5 \mathrm{~min}$. The final volume was made to the mark with mobile phase. For the sample analysis, about $500 \mathrm{mg}$ was weighed into $100 \mathrm{~mL}$ volumetric flask. Mobile phase was added up to $50 \mathrm{~mL}$ and sonicated for $5 \mathrm{~min}$. The supernatant was then diluted to mark with mobile phase. About $1 \mathrm{~g}$ of PSA was weighed into a centrifuge tube. The prepared sample $(5 \mathrm{~mL})$ was dispensed into the tube containing PSA, vortexed for $3 \mathrm{~min}$ and filtered through a $0.45 \mu \mathrm{m}$ PTFE syringe filter. Analysis was carried out by HPLC (Agilent 1100) with the diode array detector set at $275 \mathrm{~nm}$. Samples (5 or $10 \mu \mathrm{l}$ ) were injected onto a $150 \times 3.0 \mathrm{~mm}$, $2.7 \mu \mathrm{m}$ Supelco Ascentis Express Phenyl-Hexyl Column (Supelco Inc., Bellefonte, PA, USA) maintained at $25^{\circ} \mathrm{C}$ and eluted with a $90-10 \%$ isocratic of $0.1 \%$ perchloric acid and in acetonitrile at $800 \mu \mathrm{L} / \min$ over 15 minutes. Quantification was by comparison to an authentic primary standard of caffeine.

Trigonelline analysis was performed on HPLC system (Agilent 1100) equipped with PDA detector, gradient pump unit, Kinetex $2.6 \mu \mathrm{m}$ Biphenyl $100 \AA$ LC column $150 \times 4.6 \mathrm{~mm}$ (Phenomenex, Torrance, CA, USA). Samples were eluted using mobile phase of ammonium formate: acetonitrile adjusted to $\mathrm{pH} 3.0$ with formic acid and delivered at a flow rate of $1.0 \mathrm{~mL} / \mathrm{min}$. Detector was carried out at $265 \mathrm{~nm}$. The injection volume was $10 \mu \mathrm{L}$. Data acquisition and analysis were carried out using Agilent ChemStation Software, version B.04.01, 
chromatography analysis software. Trigonelline primary standard (Sigma-Aldrich, St. Louis, MO USA) was used for quantitative analysis.

\section{Clinical Study}

\section{Inclusion and Exclusion Criteria}

This clinical case study was conducted according to guidelines laid out in the Declaration of Helsinki. All procedures involving human subjects were approved by the Ethics Committee named Comité de Investigación Biomédica para el Desarrollo de Fármacos S.A. de C.V. (Zapopan, Jal, México) (study protocol No. FC-2015-N287). Ten subjects were selected to participate. They were generally healthy with ages ranging from 22 to 35 years and BMI ranging from 24.1 to $30 \mathrm{~kg} /$ $\mathrm{m}^{2}$. Exclusion criteria included acute infections, rhinitis, influenza, diagnosis of diabetes mellitus, dietary allergies. Subjects using anti-inflammatory drugs, analgesics, statins, diabetic drugs, anti-allergy medicines, multivitamins and dietary supplements were also excluded. All participants gave written, informed consent before any experimental procedure was performed.

\section{Blood Collection and sample preparation}

Study was performed by NutraClinical Inc. (Irvine, CA, USA) according to the study protocol designed by VDF FutureCeuticals, Inc. (Irvine, CA, USA). Ten subjects were included in this pilot study. Participants (6 females and 4 males) were $>19$ and $<35$ years of age, with a BMI of 29.94 $(\mathrm{SD} \pm 6.51)$. Enrolled participants were instructed to fast for $12 \mathrm{~h}$ prior to the initial blood draw. Resting subjects were given a dosage of either two empty capsules as placebo or two $500 \mathrm{mg}$ of encapsulated WCCP (for a total $1000 \mathrm{mg}$ single dose) on day 1 . All dosages were switched on day 2 relative to each subject. Subjects were given a two-day "wash-out" period prior to switching supplementations. $350 \mathrm{~mL}$ of water was administered with the capsules on each day. Blood was collected by finger puncture and placed in lithium heparin Safe-TFill@ capillary blood collection tubes (Ram Scientific Inc. Yonkers, NY, USA). The first tube, containing 50 $\mu \mathrm{L}$ of blood, was frozen immediately for ATP assays. The second tube, containing $600 \mu \mathrm{L}$ blood was used for $\mathrm{m}$-TOR and nuclear extraction from peripheral blood cells. One last tube was collected to obtain plasma. Blood samples were drawn at baseline (time zero), $60 \mathrm{~min}$ (T60), $120 \mathrm{~min}$ (T120) and $180 \mathrm{~min}$ (T180) after supplementation.

\section{Cell Lysate preparation for mTOR detection}

mTOR cell lysates were prepared from whole blood. One hundred $\mu \mathrm{L}$ of whole blood were added to $900 \mu \mathrm{L}$ of 1X cell lysis buffer containing $1 \mathrm{mM}$ PMSF into a $2.0 \mathrm{~mL}$
LoBind microtube (Eppendorf ${ }^{\circledR}$, purchased from Fisher Scientific, Inc. Pittsburgh, PA, USA). Samples were then placed in a small ice bath and sonicated for 5 minutes. After sonication, cell lysates were centrifuged at 14,000 $\mathrm{x} \mathrm{g}$ for 10 minutes at $40 \mathrm{C}$. The cell lysate supernatant (CLS) for each sample was collected and transferred into a clean, labeled $2.0 \mathrm{~mL}$ microtube and placed on ice.

\section{mTOR Protocol}

In order to determine the optical density of mTOR in each CLS sample, p-mTOR Ser 2481, p mTOR Ser 2448, and total mTOR kits from Cell Signaling Technology ${ }^{\circledR}$ (Danvers, MA, USA) were run simultaneously. One hundred $\mu \mathrm{L}$ of CLS sample was added to each assay plate. The assay protocol for each mTOR kit was followed according to the manufacturer's instructions. Total mTOR absorbance was used as reference in the analysis in order to determine the specific activity of p-mTOR (Ser 2448) and p-mTOR (Ser 2481).

\section{Protein quantification}

The protein concentration of each CLS sample was determined by the Bio-Rad DC ${ }^{\mathrm{TM}}$ Protein Assay (Hercules, CA, USA), using bovine serum albumin (BSA) (Fisher Scientific, Grand Island, NY, USA) as a standard. In order to determine the absorbance over milligram per protein of mTOR in each CLS sample, the absorbance from each mTOR assay absorbance was divided by the protein concentration.

\section{ATP Detection and Quantification}

Blood ATP concentration was determined using an ATP Assay Kit (EMD Millipore, Billerica, MS, USA) with a modification to the original method, as previously described (19). Briefly, $10 \mu \mathrm{L}$ of lysed blood were loaded onto a white plate (Corning® Fisher Scientific, Waltham, MA, USA). One hundred $\mu \mathrm{L}$ of ATP nucleotide-releasing buffer containing $1 \mu \mathrm{L}$ luciferase enzyme mix was added in each well and the plate was immediately placed on a luminometer (LMaX, Molecular Devices; Sunnyvale, CA, USA). Readings were performed during $15 \mathrm{~min}$ at 3 min intervals at $470 \mathrm{~nm}$. Relative Light Units (RLU) was recorded and ATP concentrations were determined using a standard ATP curve.

\section{Lactate and Glucose Detection}

Glucose and lactate levels were measured at collection time points $0,60,120$, and 180 minutes. Glucose was measured using an AccuChek ${ }^{\circledR}$ Compact Plus glucometer (Roche Diagnostics, Indianapolis, IN, USA). Two $\mu$ l of fresh finger blood were loaded onto an AccuChek ${ }^{\circledR}$ Testing Strip (Roche Diagnostics, Indianapolis, IN, USA) and read from the glucometer according to 
manual instructions. Blood lactate was measured using an Accutrend ${ }^{\circledR}$ Lactate Analyzer (Roche, Mannheim, Germany). Sixteen $\mu$ of fresh finger blood were loaded onto a BM Lactate Test Strip and read from the Lactate Analyzer according to manufacturer's instructions.

\section{White blood cell isolation}

Five hundred $\mu \mathrm{L}$ of whole blood collected from finger puncture as previously described were added to a $15 \mathrm{~mL}$ falcon tube containing $10 \mathrm{~mL} 1 \mathrm{X}$ Red Blood Cell Lysis buffer at room temperature (RT) $\left(22^{\circ} \mathrm{C}\right)$. After $15 \mathrm{~min}$ incubation, samples were centrifuged at $1200 \mathrm{~g}$ for 10 min. The supernatant was discarded and $10 \mathrm{~mL}$ ice cold Dulbecco's PBS (Sigma Chem. Corp.; St. Louis, MO, USA) was added followed by centrifugation as mentioned before. Supernatant was discarded and cells were snap frozen $\left(-80^{\circ} \mathrm{C}\right)$ until use.

\section{Nuclear extracts}

White blood cell (WBC) samples were thawed on an ice bath and $300 \mu \mathrm{L} 1 \mathrm{X}$ cell lysis buffer (Cell Signaling Technologies; Danvers, MA, USA) containing $1 \mathrm{mM}$ dithiothreitol (DTT) and $10 \mu \mathrm{L} / \mathrm{mL}$ protease inhibitor cocktail (Active Motif; Carlsbad, CA, USA). Samples were vortexed and incubated on an ice bath for $30 \mathrm{~min}$. Samples were subsequently centrifuged at $14,000 \mathrm{~g}$ for $30 \mathrm{~min}$ and the supernatant was recovered. Protein concentration was determined as previously described.

\section{Nrf2 Detection}

Nrf2 was determined using Trans AM® Nrf2 ELISA kits. Nuclear extract samples were loaded at $30 \mu \mathrm{g}$ protein/well. A positive control provided was used as reference. The protocol was followed as indicated by the manufacturer's instructions.

\section{Statistical Analysis}

Total mTOR, 2448-mTOR and 2481-mTOR levels were normalized using time zero as the baseline, as well as the analyses for Nrf2. Statistical analysis was performed using the commercially available GraphPad ${ }^{\circledR}$ statistical software (Graphpad Software Inc., La Jolla, CA, USA). Descriptive statistics are presented by the mean \pm standard error. Supplementations were compared at 60,120 and 180 minutes (placebo vs WCCP) within the experimental groups with baseline and between experimental groups using a one-way analysis of variance with Tukey's post hoc analysis when a significant F-ratio was observed. Statistical significance was set at P: 0.05.

\section{Results}

We identified and quantified the major phytochemicals present in WCCP. Structures of some of the major identified components are shown in Figure 1. As reported in table 1; total chlorogenic acids were the most abundant phytochemicals present in WCCP $(44.7 \pm 3.7 \mathrm{mg} / \mathrm{g})$, of which 5-O-Caffeoylquinic acid (5-CQA) showed the highest concentration $(27.9 \pm 1.7 \mathrm{mg} / \mathrm{g})$ which represents $62 \%$ of the total CGA content. Other chlorogenic acids were also detected, such as 4-O-caffeoylquinic acid (9\%), 3-O-caffeoylquinic acid $(6 \%), 3,4-\mathrm{O}$-dicaffeoylquinic acid (5\%), 3,5-O-dicaffeoylquinic acid (5\%). Caffeine and trigonelline were additionally detected $(5.2 \pm 1.2 \mathrm{mg} / \mathrm{g}$ and $8.1 \pm 1.5 \mathrm{mg} / \mathrm{g}$; respectively). Minor compounds such as procyanidin dimer, (+)-catechin and (-)-epicatechin were less abundant.

\section{Figure 1}

Structure of major hydroxycinnamates, flavan-3-ols and flavonols detected in WCCP samples

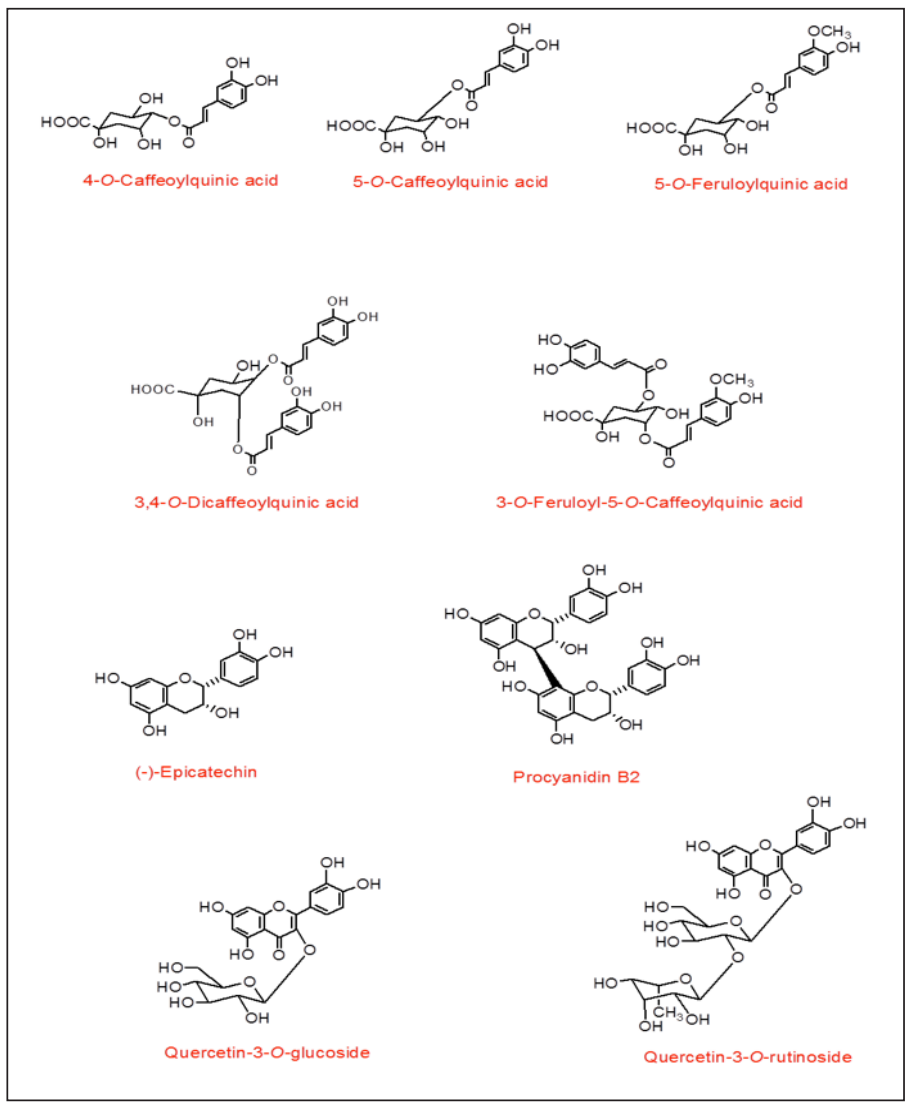

Nrf2 detected post supplementation with placebo and WCCP is shown in Figure 2. During the placebo supplementation non-significant Nrf2 increases were detected at T60 $(109 \% \pm 1 \%)$, T120 $(113 \% \pm 3 \%)$ and $\mathrm{T} 180(98 \% \pm 3 \%)$. When treated with WCCP, Nrf2 was increased at T60 $(114 \% \pm 2 \%), \mathrm{T} 120(132 \% \pm 4 \%)$ and T180 $(145 \% \pm 6 \%)$. When compared to placebo, T60 and T120 showed no significance ( $\mathrm{P}=0.5$ and $\mathrm{P}=0.3$ respectively). However, T180 was statistically significant $(\mathrm{P}=0.03)$. 
Table 1

Chemical composition of WCCP (dried whole coffee cherry powder). Results are displayed as mean $\pm S D(n=3$ true replicates $)$, in $\mathrm{mg} / \mathrm{g}$. 3-CQA

(3-O-Caffeoylquinic acid); 5-CQA (5-OCaffeoylquinic acid); 4-CQA (4-O-caffeoylquinic acid); 4-FQA

(4-O-Feruloyquinic acid); 5-FQA (5-O-Feruloyquinic acid); 3, 4-diCQA (3-4-O-Dicaffeoylquinica acid); 3,

5-diCQA (3-5-O-Dicaffeoylquinica acid); 4,5-diCQA (4-5-O-Dicaffeoylquinica acid); 3F, 4CQA (3-O-Feruloyl4-O-caffeoylquinic acid); 3C, 5FQA (3-O-Caffeoyl5-feruloyquinic acid); 4C, 5FQA (4-O-Caffeoyl-5feruloyquinic acid); CGA (Chlorogenic acid)

\begin{tabular}{lc}
\hline Major compounds (CGA), mg/g & WCCP \\
\hline 3-CQA & $3.1 \pm 0.1$ \\
5-CQA & $27.9 \pm 1.7$ \\
4-CQA & $4.2 \pm 0.2$ \\
4-FQA & $0.1 \pm 0.0$ \\
5-FQA & $3.2 \pm 0.2$ \\
3,4-diCQA & $1.3 \pm 0.1$ \\
3,5-diCQA & $2.4 \pm 0.5$ \\
4,5-diCQA & $2.2 \pm 0.0$ \\
3F,4CQA & $0.1 \pm 0.0$ \\
3C,5FQA & $0.1 \pm 0.0$ \\
4C,5FQA & $0.1 \pm 0.0$ \\
Total chlorogenic acids (CGA) & $44.7 \pm 3.7$ \\
Caffeine, mg/g & $5.2 \pm 1.2$ \\
Trigonelline, mg/g & $8.1 \pm 1.5$ \\
Minor compounds, mcg/g & $\mathrm{WCCP}$ \\
Procyanidin dimer & $12.1 \pm 0.7$ \\
(+)-catechin & $10.6 \pm 1.9$ \\
(-)-epicatechin & $7.1 \pm 1.3$ \\
Procyanidin A type trimer & $5.3 \pm 1.1$ \\
Procyanidin B type trimer & $3.0 \pm 0.5$ \\
Procynidin A type tetramer & $0.1 \pm 0.7$ \\
Procyn B type tetramer & $0.3 \pm 0.4$ \\
Quercetin-O-rutinoside & $8.9 \pm 1.6$ \\
Quercetin-3-O-rutinoside & $55.1 \pm 9.9$ \\
Quercetin-3-O-galactoside & $0.5 \pm 0.1$ \\
Quercetin-3-O-glucoside & $34.3 \pm 6.2$ \\
Total flavonols & $98.8 \pm 17.4$ \\
Total procyanidins & \\
Total minor compounds & $38.5 \pm 5.6$ \\
\hline
\end{tabular}

Glucose and lactate levels were also monitored in this 2 day study, as can be observed in Figure 3. Since subjects fasted for $12 \mathrm{~h}$ prior to the supplementations, they were monitored for possible hypoglycemia. Also, we wanted to learn whether WCCP may affect blood glucose and lactate levels. There were no observed changes for glucose or lactate on placebo or WCCP. Blood glucose was monitored for placebo (Baseline: [90.3 \pm 2.21$]$; T60:

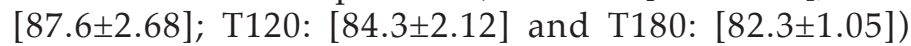

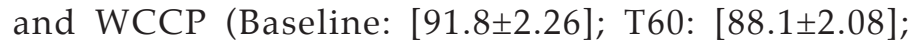

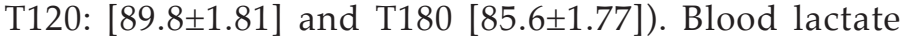
was also monitored for placebo (baseline: [0.85 \pm 0.07$]$; T60: [0.98 \pm 0.08$] ;$ T120: [0.89 \pm 0.07$]$ and T180: [0.83 \pm 0.07$])$ and for WCCP (baseline: [0.81 \pm 0.08$]$; T60: [0.83 \pm 0.09$]$; T120: [0.83 \pm 0.09$]$ and T180: [0.85 \pm 0.07$]$. It is important to reiterate that this study was conducted in healthy subjects and that any effect of WCCP has not been investigated in subjects with chronic conditions.

\section{Figure 2}

Nrf2 after supplementation with WCCP. Nrf2 was detected in nuclear extracts from isolated white blood cells. During the placebo supplementation, Nrf2 did not show any significant increase. When treated with WCCP, Nrf2 was increased at T60 (114\% $\pm 2 \%)$, T120 (132 $\pm 4 \%)$ and T180 (145\% $\pm 6 \%)$. When compared to placebo, T60 and T120 showed no significance $(\mathrm{P}=0.5$ and $\mathrm{P}=0.3$ respectively). However, T180 was significant $(\mathrm{P}=0.03)$.

Data are presented as Mean $\pm S E ; n=10$

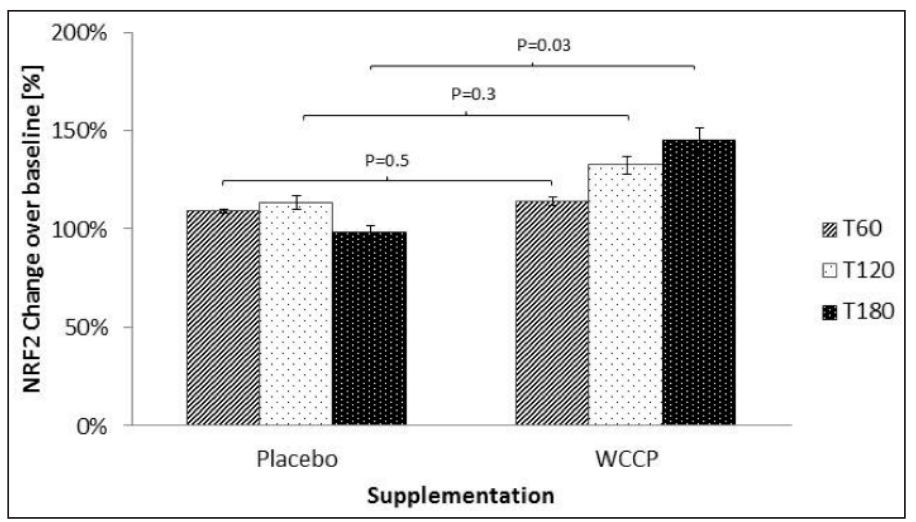

Total mTOR, 2448-mTOR and 2481-mTOR are shown in figure $4 \mathrm{~A}$. Total $\mathrm{mTOR}$ showed no changes at T60 $(107 \% \pm 7 \%), \mathrm{T} 120(98 \% \pm 6 \%)$ and $180 \mathrm{~min}(93 \% \pm 8 \%)$ in the placebo group. Supplementation with WCCP showed only as a slight non-significant change $(99 \%$ $\pm 5 \%$ at $\mathrm{T} 60 ; 107 \% \pm 9 \%$ at $\mathrm{T} 120$, and $91 \% \pm 6 \%$ at $\mathrm{T} 180)$. When compared to placebo, neither T60 ( $\mathrm{P}=0.23), \mathrm{T} 120$ $(\mathrm{P}=0.31)$ nor $\mathrm{T} 180(\mathrm{P}=0.09)$ were significant. For mTOR 2448; T60 and T180 showed no change, and for T120, there was a slight increase (13\% above baseline). For the supplemented group, T60 and T120 showed no change or significance $(\mathrm{P}=0.39 ; \mathrm{P}=0.38$, respectively) when compared to placebo. At T180, mTOR showed a decrease of $20 \%$ below baseline, which is not significant when compared to placebo $(\mathrm{P}=0.07)$ (Figure $4 \mathrm{~B})$. For mTOR 2481, placebo showed no change. Supplementation showed a non-significant reduction when compared to placebo at T60 (90\% $\pm 9 \% ; \mathrm{P}=0.22)$, T120 (97\% $\pm 14 \%$, $\mathrm{P}=0.77)$ and T180 (83\% $\pm 12 \% ; \mathrm{P}=0.19)$ (Figure 4C).

Blood ATP was detected by using a luciferasebased assay. As reported in Figure 6, ATP levels were not modified even though they showed a tendency to increase at T180. However, when compared to placebo, supplemented group was not significant at any collection point $(\mathrm{P}=0.12 ; \mathrm{P}=0.62 ; \mathrm{P}=0.5$ respectively $)$. Data are presented as Mean $\pm \mathrm{SE} ; \mathrm{n}=10$ (Figure 6). 
Figure 3

Glucose and lactate after placebo or WCCP supplementation. Subjects were fasted for $12 \mathrm{~h}$ prior to the supplementation. Both glucose and lactate were monitored at the indicated time points (T0, T60, T120 and T180), for the duration of the study. Neither glucose nor lactate showed any significant changes on either supplementation day. Data are presented as Mean $\pm \mathrm{SE}$;

$$
\mathrm{n}=10
$$

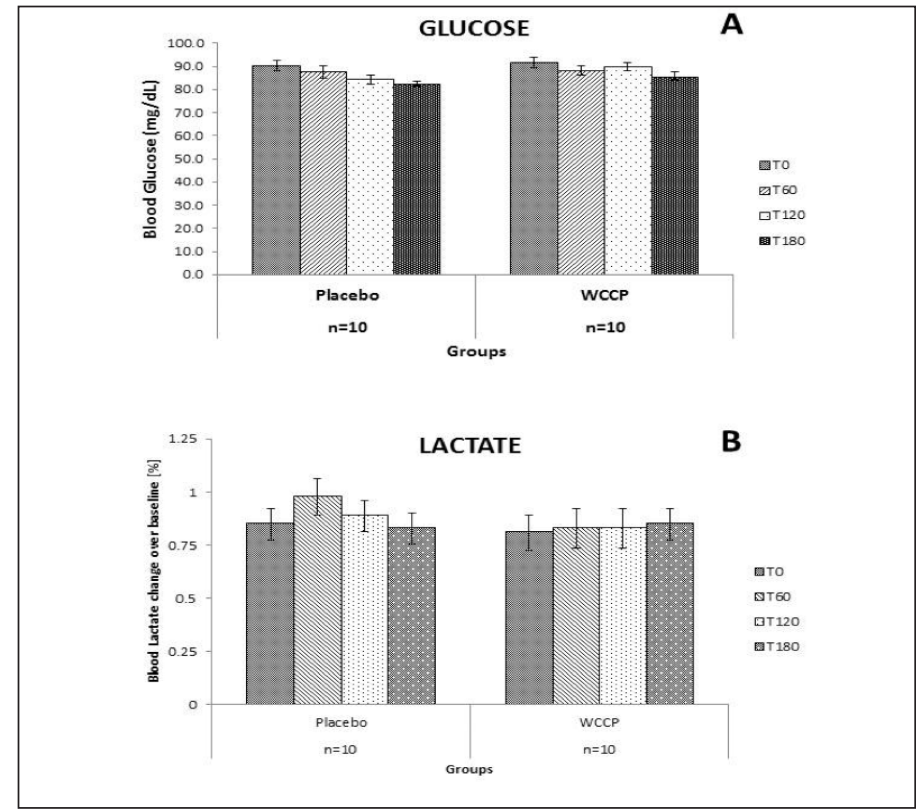

Pearson's correlation was performed for placebo NRF2 and total mTOR ( $r=0.5, \mathrm{CI}-0.88$ to $0.98 ; \mathrm{P}=0.5, \mathrm{n}=10)$, and mTOR 2481 ( $\mathrm{r}=0.3,-0.93$ to $0.97, \mathrm{P}=0.7, \mathrm{n}=10$ ) where no correlation was observed, while mTOR 2448 showed a positive correlation ( $\mathrm{r}=0.9, \mathrm{CI}-0.47$ to $0.99, \mathrm{P}=0.1, \mathrm{n}=10$ ) (Figure 5A). NRF2 and total mTOR showed a negative correlation $(\mathrm{r}=-0.3, \mathrm{CI}-0.98$ to $0.94, \mathrm{P}=0.7, \mathrm{n}=10)$, as did mTOR $2448(\mathrm{r}=-0.7, \mathrm{CI}-0.47$ to $0.99, \mathrm{P}=0.1, \mathrm{n}=10)$ and mTOR 2481 ( $\mathrm{r}=-0.7, \mathrm{CI}-0.99$ to $0.79, \mathrm{P}=0.3, \mathrm{n}=10)$.

\section{Discussion}

Dietary polyphenols such as flavones, isoflavones, flavonols, catechins and phenolic acids are mostly found in fruits and vegetables $(20,21)$. These compounds have shown antioxidant, anti-aging, anti-inflammatory, anti-atherosclerotic, and other biological abilities (11, 12). Chlorogenic acid (CGA) (5-caffeoylquinic acid) and caffeic acid, which have been reported to show antioxidant properties In vitro, $(16,20)$ are major components of coffee cherry (Table 1$)$. The chemical composition of coffee beans (green and roasted) has been studied to a considerable extent $(22,23)$, as well as the proprietary green coffee fruit $(24,25)$. Recently, the proprietary composition for two coffee fruit extracts and two coffee powders has been reported (16). In all cases, chlorogenic acids have been reported to be the most abundant components (23). In WCCP, CGA is one of the main constituents $(44.7 \mathrm{mg} / \mathrm{g})$. When compared to roasted or green coffee, green coffee fruit, coffee fruit extracts and coffee powders, WCCP has a similar composition as of that described for "coffee fruit powder" 1 and 2 (CFP-1, CFP-2) (16). For WCCP; 5-O-Caffeoylquinic acid was the main chlorogenic acid, at $62 \%$ of the total content. Other chlorogenic acids were also detected, such as 4-O-caffeoylquinic acid (9\%), 3-O-caffeoylquinic acid (6\%), 3,4-O-dicaffeoylquinic acid (5\%), 3,5-O-dicaffeoylquinic acid (5\%). Trigonelline, detected in green coffee bean (23) was also found in WCCP, as well as caffeine. Average caffeine content of regular coffee goes from $9-13 \mathrm{mg} / \mathrm{g}$ in Coffea arabiga to $15-25 \mathrm{mg} / \mathrm{g}$ in Coffea canephora (23). Whilst coffee brew contains from $90-160 \mathrm{mg}$ caffeine per cup (26), WCCP contains only $5.2 \mathrm{mg} / \mathrm{g}$.

Figure 4

mTOR detection after supplementation with placebo or WCCP. Total mTOR (A) did not show any significant changes when compared to placebo at $\mathrm{T} 60(\mathrm{P}=0.23)$, T120 ( $\mathrm{P}=0.31)$ or T180 ( $\mathrm{P}=0.09)$. mTOR $2448(\mathrm{~B})$ as well as mTOR 2481 (C) showed a reduction at T180 for the WCCP supplementation. However, when compared to placebo, neither mTOR 2448 ( $\mathrm{P}=0.07)$ nor mTOR 2481 ( $\mathrm{P}=0.19)$ were significant. Data are presented as Mean $\pm \mathrm{SE} ; \mathrm{n}=10$

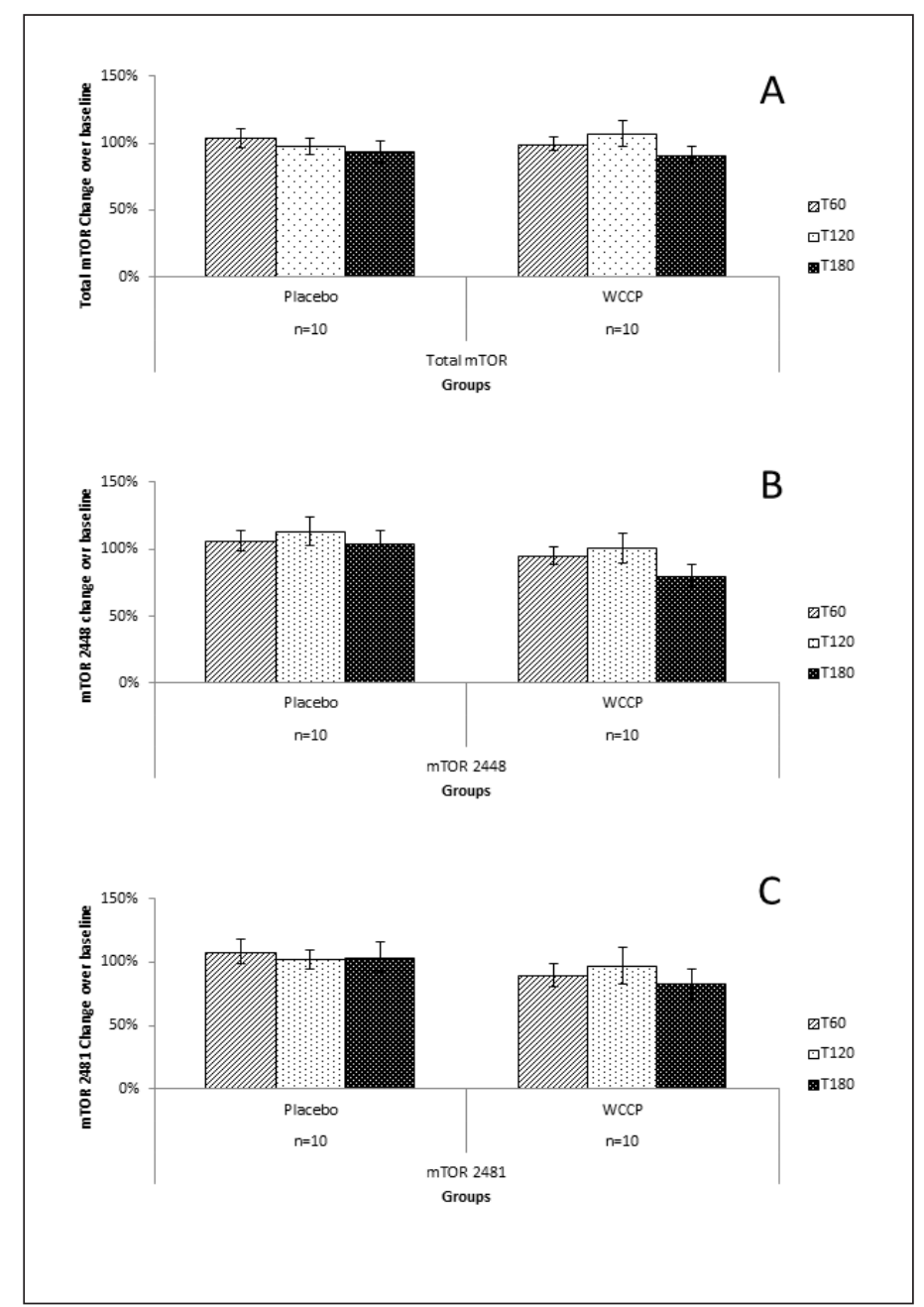




\section{Figure 5}

Correlation between Nrf2 and mTOR levels within placebo and supplemented groups. Placebo (A) Nrf2 was compared to total mTOR $(\mathrm{r}=0.43, \mathrm{n}=10)$, as well as mTOR $2448(\mathrm{r}=0.89, \mathrm{n}=10)$ and mTOR $2481(\mathrm{r}=0.27, \mathrm{n}=10)$. For the WCCP supplemented group (B), Nrf2 was also correlated with total mTOR $(\mathrm{r}=-0.32, \mathrm{n}=10)$; $\mathrm{mTOR} 2448(\mathrm{r}=-0.68$, $\mathrm{n}=10)$ and $\mathrm{mTOR} 2481(\mathrm{r}=-0.7, \mathrm{n}=10)$



Several coffee constituents such as kahweol and cafestol have been reported to increase the nuclear Nrf2 protein level and modulating ARE-mediated gene expression. More recently, chlorogenic acid has been proposed as an activator of the Nrf2/ARE pathway, activating nuclear Nrf2-translocation as well as gene expression of different phase II enzymes in the colon carcinoma cell line HT29 $(27,28)$. In a human intervention study, an increase of transcripts of phase II genes in peripheral blood lymphocytes (PBL) after 4 weeks of daily consumption of either a coffee rich in CGA or one rich in N-methylpyridinium (NMP) was observed (29).

In this study, we examined the acute effect of WCCP on peripheral blood expression of markers related to healthy aging and longevity in healthy human subjects. Acute testing was performed following a single dose, oral administration of the supplement. No significant changes in peripheral WBC Nrf2 protein levels were seen over time following consumption of placebo. In contrast, a linear increase in peripheral WBC Nrf2 protein levels was seen during the 3 hours evaluated after oral supplementation with WCCP. A significant increase from baseline control was seen at 180 minutes. Neither the effect of the placebo nor the supplementation was evaluated beyond three hours. Fasting is associated with oxidative stress and can activate Nrf2 expression (30). It has been reported that activation of Nrf2 is associated with increased glucose uptake by the pentose phosphate pathway in fibroblasts (31). Similar observations have been made in animal models (32). Cells starved of glucose have decreased Nrf2 mediated detoxification of reactive oxygen species and decreased Nrf2 initiated expression of antioxidant defense proteins (31). Diabetic animal models also suggest Nrf2 expression can affect glucose metabolism (6). In this study, baseline fasting glucose levels were in the normal range in all the subjects. Also, no changes in blood glucose or lactate levels were seen in study subjects over time following supplementation with WCCP (Figure 3). Serum glucose and lactate levels were similar to those of placebo controls. These findings suggest acute oral WCCP intake did not have a measurable impact on the pentose phosphate pathway glucose uptake during the time period examined or that the glucose uptake was compensated for by glycogenolysis or other mechanisms, even though Nrf2 expression was increased.

\section{Figure 6}

Blood ATP was measured after supplementation. ATP levels were not significantly modified. Although the trend indicates that there was a tendency to increase when compared to placebo, WCCP supplemented group was not significant at any collection point $(\mathrm{P}=0.12 ; \mathrm{P}=0.62$; $\mathrm{P}=0.5$ respectively). Data are presented as Mean $\pm \mathrm{SE}$;

$$
\mathrm{n}=10
$$

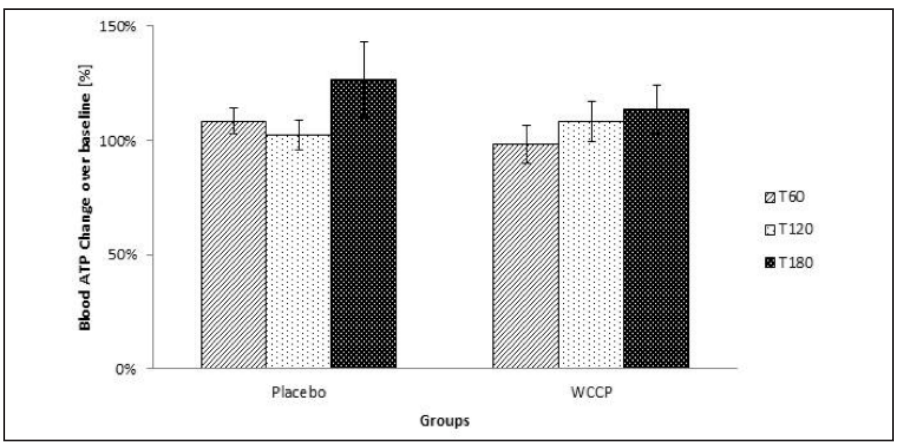

In this study, a trend towards a decrease in total mTOR S2481, and mTOR S2448 expression was seen over time (Figure 4). These findings suggest that there was minimal effect of WCCP on mTOR expression and the two related pathways. No significant correlation was found between peripheral WBC expression of Nrf2 and total mTOR, mTOR 2481, or mTOR 2448 expression after treatment with placebo, although they did appear similar. This finding suggests that the two pathways are not interlinked closely during the time period examined. The expression of Nrf2 and all three mTOR proteins appeared to be opposed, increased for Nrf2and decreased for mTOR, after supplementation with WCCP (Figure 5A, $5 B)$. These findings would be in line with current models of longevity

Activation of Nrf2 is associated with increased production of NADPH in fibroblasts (31). NADPH is responsible for providing reducing equivalents to allow glutathione or thioredoxin redox cycling, elements responsible for countering cellular redox stress. Increased NADPH synthesis is often linked to the generation of ATP, and Nrf2 expression has been correlated with ATP 
expression (33). These findings suggest increased ATP levels would be expected with increased Nrf2 expression. No significant changes in blood ATP levels were seen acutely after administration of placebo. In this study, a linear increase in blood ATP levels was seen after oral WCCP administration, although this increase was not statistically significant (Figure 6). It has been reported that cellular ATP levels were not altered in cells with genetically or pharmacologically activated Nrf-2 expression (31).

In summary, a single dose of coffee cherry powder WCCP was shown to increase Nrf2 expression. The highest Nrf2 expression was seen at the final time point of 180 minutes. Consequently, any higher subsequent expression could have been missed. Blood ATP levels remained unchanged, demonstrating that glucose is not diverted for energy production upon activation of Nrf2, whilst mTOR levels decreased. In this case, the small number of subjects evaluated may not have had enough power to detect differences in treatment outcomes. Further investigations are needed to verify whether WCCP affects Nrf2 directly or indirectly, as well as to investigate the acute and long term effect of WCCP on aging or related neurodegenerative conditions. Additional clinical testing is justified in order to further verify potency of WCCP to increase levels of Nrf2 and any potential correlation between reduced mTOR (S2448) and increased levels of Nrf2.

Funding: The present study was funded by Futureceuticals, Inc. The sponsors had no role in the design and conduct of the study; in the collection, analysis, and interpretation of data.

Conflict of interest disclosure: All authors declare that they have no conflict of interest.

Acknowledgments: We express our gratitude to John Hunter (FutureCeuticals, Inc.) for his comments and suggestions in the preparation of this paper. We would like to thank Michael Sapko for his help in editing and reviewing this manuscript.

Ethical standard: Ethical approval was granted by the Ethics Committee named Comité de Investigación Biomédica para el Desarrollo de Fármacos (Biomedical Research and Medicine Development Committee) México (Ref: FC-2015-N287).

\section{References}

1. Balligand JL, Reducing damage through Nrf-2. Cardiovasc Res, 2013. 100:1-3.

2. Gao B, Doan A and Hybertson BM, The clinical potential of influencing Nrf2 signaling in degenerative and immunological disorders. Clin Pharmacol 2014; 6:19-34.

3. Dayalan Naidu S, Kostov RV and Dinkova-Kostova AT, Transcription factors Hsf1 and Nrf2 engage in crosstalk for cytoprotection. Trends Pharmacol Sci 2015; 36:6-14.

4. Dinkova-Kostova AT and Abramov AY, The emerging role of Nrf2 in mitochondrial function. Free Radic Biol Med 2015; 88:179-88.

5. Zhang M, An C, Gao Y, et al. Emerging roles of Nrf2 and phase II antioxidant enzymes in neuroprotection. Prog Neurobiol 2013; 100:30-47.

6. Whitman SA, Long M, Wondrak GT, et al. Nrf2 modulates contractile and metabolic properties of skeletal muscle in streptozotocin-induced diabetic atrophy. Exp Cell Res 2013; 319:2673-2683.

7. Sahin K, Orhan C, Tuzcu M, et al. Orally administered lycopene attenuates diethylnitrosamine-induced hepatocarcinogenesis in rats by modulating $\mathrm{Nrf}-2$ HO-1 and Akt/mTOR pathways. Nutr Cancer 2014; 66:590-8.

8. Harrison DE, Strong R, Sharp ZD, et al. Rapamycin fed late in life extends lifespan in genetically heterogeneous mice. Nature 2009; 460:392-5.

9. Johnson SC, Rabinovitch PS and Kaeberlein M, mTOR is a key modulator of ageing and age-related disease. Nature 2013; 493: 338-45.

10. Papaioannou AI, Rossios C, Kostikas K, et al. Can we delay the accelerated lung aging in COPD? Anti-aging molecules and interventions. Curr Drug Targets 2013; 14:149-57.

11. Kishimoto Y, Tani M and Kondo K, Pleiotropic preventive effects of dietary polyphenols in cardiovascular diseases. Eur J Clin Nutr 2013; 67:532-535.

12. Tomás-Barberán FA and Andrés-Lacueva C. Polyphenols and Health: Current State and Progress. J. Agric. Food Chem 2012; 60:8773-8775.

13. Dinkova-Kostova AT, Talalay P. Direct and indirect antioxidant properties of inducers of cytoprotective proteins. Mol Nutr Food Res 2008; 52:S128-38.

14. Jeong WS, Jun M and Kong AN, Nrf2: a potential molecular target for cance chemoprevention by natural compounds. Antioxid Redox Signal 2006; 8:99-106.

15. Nicoli, MC, Anese M, Manzocco L, et al. Antioxidant properties of coffee brews in relation to the roasting degree. Lebensm Wiss Technol 1997; 30:292-297.

16. Mullen W, Nemzer B, Ou B, et al. The antioxidant and chlorogenic acid profiles of whole coffee fruits are influenced by the extraction procedures. J Agric Food Chem 2011; 59:3754-62.

17. Mullen W, Nemzer B, Stalmach A, et al. Polyphenolic and hydroxycinnamate contents of whole coffee fruits from China, India, and Mexico. J Agric Food Chem 2013; 61:5298-309.

18. Duarte GS, Pereira AA and Farah A. Chlorogenic acids and other relevant compounds in Brazilian coffees processed by semi-dry and wet post-harvesting methods. Food Chemistry 2010; 118:851-855.

19. Reyes-Izquierdo T, Hammond, LE; Sikorski, RS; et al. Acute effect of HH2o on oxygen consumption rate, intracellular ATP and ROS in freshly isolated human peripheral blood mononuclear cells. Current Topics in Nutraceutical Research 2011; 9:139-146.

20. Han X, Shen T, Lou H. Dietary polyphenols and their biological significance. Int J Mol Sci 2007; 8:950-988.

21. Kim HS, Quon MJ and Kim JA, New insights into the mechanisms of polyphenols beyond antioxidant properties; lessons from the green tea polyphenol, epigallocatechin 3-gallate. Redox Biol 2014; 2:187-95.

22. Crozier A, Jaganath IB and Clifford MN, Dietary phenolics: chemistry, bioavailability and effects on health. Natural Product Reports 2009; 26:1001-1043.

23. Chu YF, Coffee: emerging health effects and disease prevention. 2012. John Wiley \& Sons.

24. Farah A, Monteiro M, Donangelo CM, et al. Chlorogenic acids from green coffee extract are highly bioavailable in humans. J Nutr 2008; 138:2309-15.

25. Farah A, Donangelo CM. Phenolic compounds in coffee. Brazilian Journal of Plant Physiology, 2006. 18:23-36.

26. Blauch JL and Tarka SM, HPLC determination of caffeine and theobromine in coffee, tea, and instant hot cocoa mixes. J Food Sci 1983; 48:745-747.

27. Boettler U, Volz, N., Pahlke, G., et al. Coffees rich in chlorogenic acid or $\mathrm{N}$-methylpyridinium induce chemopreventive phase II-enzymes via the Nrf2/ ARE pathway in vitro and in vivo. . Mol. Nutr Food Res 2011; 55:798-802.

28. Boettler U, Volz N, Pahlke G, et al. Coffee constituents as modulators of Nrf nuclear translocation and ARE (EpRE)-dependent gene expression. J Nutr Biochem 2011; 22:426-440.

29. Volz N, Boettler U, Winkler S, et al. Effect of coffee combining green coffee bean constituents with typical roasting products on the Nrf2/ARE pathway in vitro and in vivo. J Agric Food Chem 2012; 60:9631-41.

30. Zhang YK, Wu KC and Klaassen CD, Genetic activation of Nrf2 protects against fasting-induced oxidative stress in livers of mice. PLoS One 2013; 8:e59122.

31. Heiss EH, Schachner D, Zimmermann K, et al. Glucose availability is a decisive factor for Nrf2-mediated gene expression. Redox Biol 2013; 1:359-365.

32. Bardoux P, Zhang P, Flamez D, et al. Essential role of chicken ovalbumin upstream promoter-transcription factor II in insulin secretion and insulin sensitivity revealed by conditional gene knockout. Diabetes 2005; 54: 1357-63.

33. Holmstrom KM, Baird L, Zhang Y, et al. Nrf2 impacts cellular bioenergetics by controlling substrate availability for mitochondrial respiration. Biol Open 2013; 2:761-770. 